BASIC SCIENCE REVIEW

Robert L. Ferris, MD, PhD, Section Editor

\title{
CANCER CACHEXIA SYNDROME IN HEAD AND NECK CANCER PATIENTS: PART I. DIAGNOSIS, IMPACT ON QUALITY OF LIFE AND SURVIVAL, AND TREATMENT
}

\author{
Marion Couch, MD, PhD, ${ }^{1,6}$ Victor Lai, MS, ${ }^{2}$ Trinitia Cannon, MD, ${ }^{1}$ Denis Guttridge, $\mathrm{PhD},{ }^{3}$ \\ Adam Zanation, MD, ${ }^{1}$ Jonathan George, BA, ${ }^{2}$ D. Neil Hayes, MD, ${ }^{4,6}$ Steven Zeisel, MD, PhD, ${ }^{5}$ \\ Carol Shores, MD, PhD ${ }^{1,6}$ \\ ${ }^{1}$ Department of Otolaryngology/Head and Neck Surgery, University of North Carolina School of Medicine, \\ G0412 Neurosciences Hospital, Chapel Hill, NC 27599-7070. E-mail: marion-couch@med.unc.edu \\ ${ }^{2}$ Verne S. Caviness General Clinical Research Center, University of North Carolina School of Medicine, \\ G0412 Neurosciences Hospital, Chapel Hill, North Carolina \\ ${ }^{3}$ Division of Human Genetics, Department of Molecular Virology, Immunology and Medical Genetics, \\ Arthur G. James Comprehensive Cancer Center, The Ohio State University, Columbus, Ohio \\ ${ }^{4}$ Division of Medical Oncology, Department of Internal Medicine, University of North Carolina School of \\ Medicine, Chapel Hill, North Carolina \\ ${ }^{5}$ Department of Nutrition, School of Public Health, Department of Pediatrics, University of North Carolina \\ School of Medicine, Chapel Hill, North Carolina \\ ${ }^{6}$ Lineberger Comprehensive Cancer Center, University of North Carolina School of Medicine, \\ Chapel Hill, North Carolina
}

Accepted 23 January 2006

Published online 6 February 2007 in Wiley InterScience (www.interscience.wiley.com). DOI: 10.1002/hed.20447

\begin{abstract}
Background. Cancer cachexia is a debilitating, wasting condition that affects many cancer patients, including those with head and neck cancer. The overall incidence of cancer cachexia is quite high for some types of cancer, and cachexia will be the main cause of death for more than $20 \%$ of all cancer patients. This syndrome uniquely challenges patients with head and neck cancer. This article outlines the diagnosis of cancer cachexia, reviews its impact on patient quality of life (QOL) and survival, and updates the reader on potential therapies that may suppress it.
\end{abstract}

\section{Correspondence to: M. Couch}

Contract grant sponsors: Supported by grant RR000046 from the University of North Carolina General Clinical Research Center; and the Doris Duke Clinical Research Fellowship Program.

๑) 2007 Wiley Periodicals, Inc.
Methods. A comprehensive literature search was performed using PubMed of the National Library of Medicine, which includes more than 15 million citations back to the 1950s. The Cochrane Library and Google search engine were used as well.

Results. This syndrome differs significantly from starvation, and thus accurate and timely diagnosis is essential. Nutritional therapy alone is insufficient. Current management strategies include corticosteroids and megesterol acetate, in conjunction with nutritional therapy. Future strategies may include nutraceuticals, omega-3 fatty acids, inflammatory antagonists, and other targeted treatments.

Conclusions. Because cancer cachexia differs significantly from starvation, nutritional supplementation must be used in conjunction with other anti-cachexia agents to reverse the chronic systemic inflammatory state and the effects of circulating tumorderived factors seen in cachexia. Careful identification of patients at risk and those suffering from this syndrome will lead 
to better outcomes and treatments. Ultimately, more research is needed to better treat this devastating condition.

(C) 2007 Wiley Periodicals, Inc. Head Neck 29: 401-411, 2007

Keywords: cancer cachexia and anorexia; muscle wasting, weight loss

The true incidence of cachexia in patients with head and neck squamous cell carcinoma (HNSCC) is unknown, but more than $50 \%$ of patients with advanced head and neck cancer have significant weight loss and possible cachexia. ${ }^{1,2}$ More than $80 \%$ of pancreatic cancer patients and $60 \%$ of lung cancer patients present at diagnosis with cachexia. $^{3,4}$ This wasting syndrome should be suspected in any cancer patient with an involuntary weight loss of greater than $5 \%$ of the premorbid weight within the previous 6-month period. ${ }^{5}$ Patients with cachexia will have generalized asthenia, marked unintentional weight loss, sarcopenia, anemia, anorexia, and emotional and mental fatigue, all of which cause a general decline in functional status. These patients report a marked reduction in their quality of life (QOL). ${ }^{6,7}$ Prognosis is adversely affected and response to therapy may be diminished with increased complications and toxicities to treatment seen in these patients. ${ }^{8-10}$

Overall, cachexia accounts for $20 \%$ of all cancerrelated deaths. ${ }^{11}$ Death may occur when the patient loses approximately $30 \%$ of premorbid weight due to generalized weakness, immobility, and loss of respiratory muscle function. However, loss of as little as $5 \%$ body weight may worsen the patient's prognosis. ${ }^{12}$ Furthermore, weight loss is an independent predictor of both prognosis and survival in the cachectic cancer patient. ${ }^{8,9}$ The rate of weight loss and the total weight loss are directly related to the survival of the cancer patient. ${ }^{12}$ This is likely because cancer cachexia is associated predominantly with skeletal muscle loss, necessary for ambulation and adequate pulmonary function, as compared with primarily adipose tissue loss seen in starvation.

Cancer cachexia and starvation are distinctly different. Starvation involves conservation of lean body mass (LBM) and preferential increase in fat metabolism in the liver to supply energy. Ketone bodies, which are derived from this fat metabolism, provide an important energy source for the brain. Also, resting energy expenditure (REE) is lowered in starving patients, which matches these patients' reduced energy intake. Cancer cachexia appears to be a very different phenomenon. It involves preferential loss of skeletal muscle over
Table 1. Cancer cachexia differs from starvation.

\begin{tabular}{lcc}
\hline Parameter & Cachexia & Starvation \\
\hline Resting energy expenditure & ++ & - \\
Loss of skeletal muscle & ++ & - \\
Loss of fat & ++ & ++ \\
Loss of visceral muscle & - & + \\
Acute-phase response & ++ & - \\
Proinflammatory cytokines & ++ & - \\
Toxohormones & ++ & - \\
Increased liver metabolism & ++ & - \\
Liver size & ++ & -- \\
\hline
\end{tabular}

Abbreviations: + , increased; -, reduced.

adipose tissue, increased proteolysis and lipolysis, increased metabolic activity of the liver, and increased production of acute-phase proteins (Table 1). Cancer cachexia involves inappropriate elevation of REE in many tumor types, even as energy intake may be reduced due to anorexia. ${ }^{3} \mathrm{In}$ contrast to starvation, both LBM and fat are dramatically reduced, visceral muscle protein is spared, and skeletal muscle protein is lost. ${ }^{3}$

Tumors will lead to the production of lactate, which will be converted back to glucose in the liver, but at a tremendous expense of energy, using the inefficient Cori cycle (Figure 1). The liver becomes enlarged as a result of the increased metabolism of glucose, but also due to the production of acutephase proteins such as C-reactive protein (CRP) and fibrinogen, which are elevated in patients with cachexia. Proinflammatory cytokines, secreted by either the tumor or the host, initiate an exuberant systemic inflammatory response, which also results in an increased production of acute-phase proteins in the liver, further depleting essential amino acids. As mentioned above, the loss of skeletal muscle, or sarcopenia, causes significant impairment and physiologic weakness in the patient. Proinflammatory cytokines such as tumor necrosis factor- $\alpha$ (TNF- $\alpha$ ), interleukin-6 (IL-6), interleukin$1 \beta$ (IL-1 $\beta$ ), and interferon- $\gamma$ (IFN- $\gamma$ ) are implicated because of their ability to cause muscle wasting. ${ }^{13,14} \mathrm{~A}$ more complete review of this topic will be provided in "Cancer Cachexia Syndrome in Head and Neck Cancer Patients: Part II. Pathophysiology."

The pathogenesis of cancer cachexia is not strictly a function of tumor burden, as patients with small tumor volumes are often diagnosed with cachexia, whereas this syndrome may never develop in patients with enormous tumor burdens. Despite this, cachexia does appear frequently in patients with advanced stages of can- 


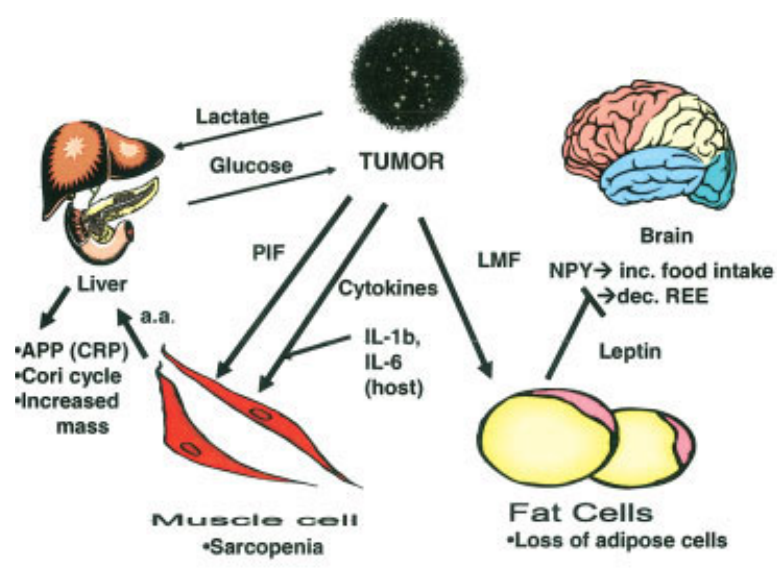

Abbreviations: a.a. - amino acids, APP - acute phase proteins, CRP. Creactive proteins, inc. - increase, dec. - decrease, REE - resting energy expenditure, TNF- tumor necrosis factor- $\alpha$, LMF - lipid mobilizing factor, IL-1b interleukin 1b, 6 - interleukin-6, PIF - proteolysis inducing factor, NPY neuropeptide $Y$.

FIGURE 1. Clinical manifestations of cancer cachexia. Toxohormones such as lipid-mobilizing factor (LMF) and proteolysisinducing factor (PIF) along with proinflammatory cytokines are secreted by the tumor cells to promote proteolysis of skeletal muscles and lipolysis of body fat. Amino acids from the protein catabolism are transported to the liver, where liver mass increases due to the increased metabolism. The liver converts lactate to glucose using the energy-inefficient Cori cycle. An acute-phase response is seen with production of acute-phase proteins such as C-reactive protein (CRP). This limits the availability of critical amino acids for protein synthesis of new muscle. Disruption of the leptin regulation and neuropeptide $Y$ (NPY) regulation lead to inappropriate increase in the resting energy expenditure (REE) and decreased stimulation of feeding. [Color figure can be viewed in the online issue, which is available at www.interscience.wiley.com.]

cer. This may be explained by tumor-derived factors called toxohormones that induce cancer cachexia in animal models and which are detected in the urine of cachectic patients. ${ }^{15}$ Lipid-mobilizing factor (LMF), a prominent toxohormone in cancer cachexia, is identical to the human plasma protein $\mathrm{Zn}-\alpha$-2-glycoprotein (ZAG). ${ }^{15}$ It acts through a $\beta_{3}$-adrenoceptor to mediate the lipolysis of adipose tissue. Another toxohormone, the tumor-derived proteolysis-inducing factor (PIF), is a $24-\mathrm{kDa}$ sulfated glycoprotein capable of inducing cachexia (Figure 1). PIF induces IL-6 production, inhibits protein synthesis, and stimulates protein breakdown through an activation of nuclear factor kappa B (NF- $\mathrm{kB})$ that further upregulates the ubiquitin proteasome system. ${ }^{15}$

Cancer cachexia often involves anorexia, but this alone does not fully explain the wasting that is seen. There appears to be a dysregulation of the adaptive feeding response in cachexia that involves leptin, a hormone secreted by adipose tis- sue that functions to regulate feeding and energy expenditure in starvation. ${ }^{16}$ Low levels of leptin are seen in weight loss, which stimulates feeding through increased hypothalamic orexigenic signaling. ${ }^{3,5}$ In cancer cachexia, however, leptin levels are inappropriately altered. One proposed mechanism suggests that increased leptin release disrupts the normal regulation of food intake and energy expenditure. This inhibits the regulatory activity of leptin and serves to decrease food intake and to increase REE. ${ }^{5}$ Interestingly, neuropeptide Y (NPY), a potent feeding-stimulatory peptide in the same regulatory pathway as leptin, is also dysregulated in cancer cachexia. Experimental evidence in animals suggests that NPY levels are reduced in tumor-bearing mice. ${ }^{17}$ The cytokine IL-1 decreases NPY levels in the hypothalamus, suggesting that this cytokine may act centrally to contribute to the development of cachexia. ${ }^{18}$

Although more research is needed regarding the pathogenesis of cancer cachexia, it is clear that this syndrome is distinctly different from simple starvation, and that there appear to be multiple etiologies affecting multiple end organs.

\section{DIAGNOSIS}

The diagnosis of cancer cachexia is complex and multifaceted and requires meticulous clinical examination of the patient (Table 2). In ascertaining the patient history, the presence of unintentional weight loss of more than $5 \%$ of premorbid weight in a 6 -month period should be noted. This may require careful questioning of the patient about appetite, satiety, food intake, and dysphagia. The presence of anorexia should also be ascertained, as it is commonly associated with cancer cachexia and will contribute to decreased energy intake. Recent chemotherapy or radiation treatment should be considered, as treatment-related toxicities may worsen anorexia and/or cachexia (Table 3). Last, the patient should be queried about increased fatigue and emotional distress, both of which may be associated with the loss in QOL and functional status seen in cancer cachexia.

Physical examination may reveal skeletal muscle wasting, loss of body fat, and generalized weakness. Although most clinicians depend on body weight as a general measure of nutritional status, skinfold thickness, and arm muscle circumference and area may also be used to assess patients. ${ }^{5}$ It is important to examine such muscles as the gastrocnemius, vastus lateralis, rectus abdominus, and 


\begin{tabular}{|c|c|}
\hline Test & Finding \\
\hline \multicolumn{2}{|l|}{ Clinical } \\
\hline Body weight & $\begin{array}{c}\text { Unintentional weight loss ( }>5 \% \\
\text { during preceding } 6 \text { months) }\end{array}$ \\
\hline Skeletal muscle mass & $\begin{array}{l}\text { Decreased biceps, quadriceps } \\
\text { muscle mass }\end{array}$ \\
\hline Food intake recall or diary & $\begin{array}{l}\text { Anorexia and/or decreased } \\
\text { food intake }\end{array}$ \\
\hline Fatigue & Increased \\
\hline Range of motion & Usually impaired \\
\hline Quality-of-life surveys & Decreased scores \\
\hline $\begin{array}{l}\text { Karnofsky } \\
\text { Performance Scale }\end{array}$ & Decreased scores \\
\hline \multicolumn{2}{|l|}{ Serum: } \\
\hline Serum CRP & $\begin{array}{l}\text { Increased } \\
\text { (acute-phase response) }\end{array}$ \\
\hline Serum fibrinogen & $\begin{array}{l}\text { Increased } \\
\text { (acute-phase response) }\end{array}$ \\
\hline Serum hematocrit & Decreased (anemia) \\
\hline Serum albumin & Decreased \\
\hline \multicolumn{2}{|l|}{ Nutritional assessment } \\
\hline Indirect calorimetry & Increase in REE \\
\hline DXA & Decrease in LBM \\
\hline
\end{tabular}

Abbreviations: CRP, C-reactive protein; REE, resting energy expenditure; DXA, dual X-ray absorptimetry; LBM, lean body mass.

biceps, as experimental data and clinical observations suggest that these type II fast-twitch muscles are most commonly affected in cancer cachexia. ${ }^{19-22}$ Involuntary weight loss in combination with loss of LBM should alert the clinician to the strong possibility of cancer cachexia.

Screening cancer patients for cachexia has included obtaining blood to detect low serum albumin, low hematocrit, and fibrinogen levels, but these laboratory values are quite nonspecific for nutritional evaluation. Measurement of short half-life proteins (eg, transferrin and transthyretin) and urinary metabolites of protein breakdown (eg, creatinine) are of limited value, as they may be elevated in cancer patients with chronic malnutrition but in those with not cachexia. ${ }^{5}$ Increased acute-phase proteins such as CRP levels may be an especially helpful screening test, as elevated CRP has been positively correlated with weight loss and cancer cachexia. ${ }^{23}$ Although relatively nonspecific, CRP is a sensitive marker for inflammation that is both inexpensive and routinely available, making it useful in the diagnosis of cancer cachexia.

Sophisticated analytical techniques may be used to diagnose cancer cachexia. For example, dual energy X-ray absorptiometry (DXA) scans and bioelectrical impedance analysis can assist in determining patient body composition, which is altered in cachexia. DXA uses alternating highenergy and low-energy X-rays to analyze the differences between bone and soft tissue attenuation at different X-ray levels. It can be used to determine the relative proportions of LBM, fat body mass, and total body water (TBW), and exposes each patient to less radiation than computed tomography (CT). In cancer patients with cachexia, both LBM and fat body mass will be decreased. Bioelectrical impedance analysis can assess both the nutritional status and the fluid deficits in patients with advanced cancer and is based on the principle that body tissues differentially oppose the flow of a small alternating current. ${ }^{24}$

Indirect calorimetry, another diagnostic tool in cancer cachexia, permits the assessment of REE and is an accurate and clinically feasible method of measuring energy expenditure. Because of the direct relationship between caloric burn and oxygen consumption, measuring the volume of oxygen uptake $\left(\mathrm{V}_{\mathrm{O} 2}\right)$ will elucidate a patient's caloric burn rate. Measurements of the oxygen consumption rate and the carbon dioxide production rate can be used to calculate the patient's respiratory quotient and metabolic rate.

\begin{tabular}{ll}
$\begin{array}{c}\text { Table 3. Possible treatment-related toxicities contributing to } \\
\text { cancer cachexia in patients with head and neck cancer. }\end{array}$ \\
\hline Treatment modality & \multicolumn{1}{c}{ Treatment-related toxicity } \\
\hline Surgery & Mechanical interference in digestive tract \\
& Dysphagia \\
& Poorly fitting dentures \\
& Loss of dentition \\
& Odynophagia \\
& Psychological distress \\
& Depression \\
& Thyroid insufficiency \\
& Nausea \\
& Mucositis \\
& Erosive lesions in digestive tract \\
& Taste alterations \\
& Xerostomia \\
Radiation therapy & Thrush \\
& Esophageal strictures \\
& Fatigue \\
& Thyroid insufficiency \\
& Nausea \\
Mucositis & Erosive lesions in digestive tract \\
Thrush & Taste alterations \\
Diarrhea \\
Vomiting \\
Abdominal cramping and bloating \\
Fatigue \\
Learned aversions to specific foods \\
\\
Chemotherapy
\end{tabular}


These measurements permit more accurate monitoring of patients, especially if treatment of the cachexia is initiated.

\section{IMPACT ON QUALITY OF LIFE AND SURVIVAL}

Cancer cachexia has a detrimental effect on patient QOL and survival. It is associated with increased fatigue and emotional distress, which is reflected in decreased performance status and QOL indices. ${ }^{6,9}$ Current evidence supports treating cachexia, as it may improve the quality of these patients' lives. ${ }^{6,7}$ In particular, improvement in the nutritional status of cachectic cancer patients appears to be associated with increased QOL scores, increased Karnofsky performance status, and increased LBM. ${ }^{25}$ In a recent study of patients with advanced-stage HNSCC undergoing radiation or chemoradiation therapy, pretreatment fatigue levels were inversely correlated with survival. ${ }^{26}$ Two QOL questionnaires were given to more than 100 patients, and an increase in the baseline fatigue score of 10 points was associated with a $17 \%$ reduction in the likelihood of survival. Because cancer cachexia causes fatigue, it may be reasonable to investigate this syndrome further as the cause of the reduction in survival. In another recent study of patients with HNSCC, those with weight loss of greater than $10 \%$ during treatment had worse health-related QOL scores, and the reduced scores persisted even for more than 1 year. In addition, the 3-year survival rate was worse for the group with greater than $10 \%$ weight loss. ${ }^{27}$ Further investigation may clarify the relationship between cachexia, fatigue, and survival in HNSCC patients. This might allow for a deeper understanding of how cancer cachexia impairs QOL and decreases survival in these patients.

In a landmark study, Dewys ${ }^{10}$ assessed the prognostic effect of weight loss before chemotherapy. Cancer patients with cachexia-induced weight loss ( $\geq 5 \%$ body weight lost involuntarily) experienced shorter median survival times than cancer patients without weight loss. Furthermore, the patients with weight loss had poorer responses to chemotherapy and experienced more treatment toxicities. ${ }^{10}$ In another study investigating whether weight loss influenced the outcome of patients undergoing chemotherapy for gastrointestinal carcinomas, cachectic patients experienced decreased survival, decreased treatment response, shorter disease-free intervals, and decreased performance status compared with patients without weight loss at presentation. ${ }^{9}$

Cachexia can have a significant effect on patients with small tumor burdens. In a recent study of early-stage (T1N0M0) renal cell carcinoma patients, the presence of cachexia was associated with markedly worse disease-specific survival. The 5-year survival rate in patients with high-grade (3 or 4) tumors, with and without cachexia, was $55 \%$ and $75 \%$, respectively. ${ }^{28}$

With regard to patients with HNSCC, weight loss of greater than $10 \%$ had a strong prognostic impact on 1-year survival and could be used to predict mortality after recurrent oral cavity and oropharyngeal carcinomas in a retrospective review. $^{29}$ Survival was poorest in patients with greater than $10 \%$ weight loss at the time of recurrence and best for patients with no weight loss. ${ }^{29}$

The above studies indicate that cancer cachexia is associated with both decreased QOL and survival in patients with cancer, including those with head and neck cancer. Further studies are needed to clarify the extent to which weight loss impacts QOL and survival in the cachectic cancer patient.

\section{UNIQUE CHALLENGES FOR PATIENTS WITH HEAD AND NECK CANCER}

Head and neck cancer cachexia presents several unique challenges, as patients with head and neck cancer are often at risk of multifactorial malnutrition. First, the site of the primary tumor may mechanically obstruct the aerodigestive tract or cause odynophagia or dysphagia. Second, head and neck cancer patients may have underlying chronic malnutrition at presentation due to alcohol and tobacco abuse and poor nutritional habits. Finally, treatment-related toxicities, such as radiation exposure to the aerodigestive tract and chemotherapeutic agents, may further impair the patient's ability to obtain adequate nutrition due to mucositis or xerostomia (Table 3 ).

Of all patients who undergo radiation therapy, head and neck cancer patients appear to be at highest risk of malnutrition. ${ }^{7}$ One study found that $52 \%$ of head and neck cancer patients were malnourished at the onset of radiation therapy. Importantly, the incidence of odynophagia, dysphagia, anorexia, and nausea increased after these patients received radiotherapy. In this same study, poorer nutritional status and intake of food were associated with decreased mobility, limited activity, and increased depression and anxiety. 
When nutritional support was initiated, improvements in patient nutritional intake were correlated with improvements in QOL scores. This supports the role of nutritional counseling in improving the head and neck cancer patients' QOL. ${ }^{7}$

Mucositis is common in patients undergoing combined chemoradiation treatment and is frequently severe enough to require treatment interruptions or delays. Xerostomia is also common after radiation and frequently will not resolve after treatment. A recent study of oropharyngeal cancer patients revealed that reduction in saliva production continued for well over 1 year after chemoradiation therapy. ${ }^{30}$ Although the reduction of saliva production observed in this study did not correlate with inefficient swallowing, it did change patients' perceptions of their ability to swallow. In a patient with cancer cachexia, xerostomia or altered taste perception may predispose the patient to decreased nutritional intake for many months after treatment. This further worsens the prognosis of the cachectic cancer patient. ${ }^{30}$

In a prospective study looking at the incidence of weight loss in 100 consecutive head and neck cancer patients undergoing radiation therapy, $57 \%$ of patients lost weight during treatment, with a mean weight loss of $6.5 \mathrm{~kg}$ (approximately $10 \%$ of their body weight). A questionnaire to determine causative factors revealed that dry mouth, poorly fitting dentures, and sore mouth were most frequently cited. Change in taste perception, metallic taste, loss of taste, nausea, and constipation were also frequently cited. ${ }^{1}$

Although important, food intake alone does not appear to correlate with the degree of malnutrition seen in cachectic cancer patients. For this reason, attempts to increase food intake may fail to fully reverse progressive weight loss unless other forms of anti-cachexia treatment are initiated. ${ }^{31}$ As the cancer cachexia seen in head and neck cancer patients is similar to that in patients with other types of cancers, improved diagnosis and treatment should benefit head and neck cancer patients by improving their QOL, response to therapy, and survival.

\section{TREATMENT}

Cachexia treatment should incorporate increasing nutritional intake with agents that will inhibit muscle and fat wasting and possibly reduce the patient's inflammatory response. Glucocorticosteroids are a commonly used class of agents that have shown short-term improvement in performance status and sense of well-being, as well as increased appetite and food consumption, but not increased body weight. ${ }^{5}$ Appetite is improved and pain and nausea are better controlled with glucocorticosteroids. However, such improved symptom control is likely only a temporary effect, as recent studies have suggested that glucocorticosteroids may promote lipolysis and increase production of the tumor toxohormone ZAG (or LMF). ${ }^{32}$ Dexamethasone, which exhibits the least amount of mineralocorticoid activity, has been identified as the steroid of choice in the treatment of cachexia. ${ }^{33}$ It may also be an appropriate choice for use in patients with limited life expectancy. Side effects may be significant, however, especially with prolonged use (4-6 weeks) and may include osteoporosis, proximal muscle weakness, immunosuppression, and delirium. Indeed, dexamethasone may itself induce skeletal muscle atrophy. ${ }^{34}$

Perhaps the most potent appetite stimulant, or orexigenic agent, is megestrol acetate (Megace, Bristol-Myers Squibb, New York, NY). This synthetic hormone, called progestogen, can mimic the actions of progesterone and interfere with hormone signaling in the body. High-dose therapy with megestrol or medroxyprogesterone improves appetite and the QOL for many cachectic cancer patients, but there has been no documented effect on LBM or change in performance status (Table 4). Megestrol acetate can induce weight gain, but body composition studies show increases in increased body fluid and adipose tissue rather than LBM. ${ }^{35}$ Weight gain has been confirmed in some studies of megestrol acetate use in head and neck cancer patients, but it is not clear whether any effect on LBM is present. For instance, megestrol acetate $(320 \mathrm{mg} /$ day) was given to male patients with head and neck cancer during 3 chemotherapy cycles. ${ }^{36}$ The average weight gain was $6.3 \mathrm{~kg}$, but body composition studies were not performed, so the effect on LBM is unknown. However, increases were seen in patient appetite and QOL score, whereas decreases were seen in serum levels of IL-1, IL-6, and TNF- $\alpha$ in patients after treatment.

In another study, 38 patients were treated with $160 \mathrm{mg} /$ day of megestrol acetate for 4 months. Significant weight gains were seen after 8 weeks with a mean weight gain of $4.58 \pm 3.19 \mathrm{~kg}$. ${ }^{2}$ In a study of 64 patients with head and neck cancer who were undergoing intensive chemoradiation, $160 \mathrm{mg} /$ day of megestrol acetate was given during and for 6 weeks after treatment. Patients receiving megestrol acetate did not experience deterio- 


\begin{tabular}{|c|c|c|}
\hline Treatment mode & Dose & Mechanism of action \\
\hline \multicolumn{3}{|l|}{ Corticosteroids* } \\
\hline Dexamethasone & 3-6 mg po QD & Improve appetite, improve pain control, \\
\hline Prednisolone & 5 mg po TID & antiemetic effect, but no beneficial \\
\hline Methylprednisolone & 125 mg IV QD & effect on LBM \\
\hline \multicolumn{3}{|l|}{ Progestational drugs ${ }^{\dagger}$} \\
\hline Megestrol & 40-400 mg po QID & Improve appetite and caloric intake, weight \\
\hline Medroxyprogesterone & 500 mg po BID & gain is mostly fat, not LBM \\
\hline $\begin{array}{l}\text { Nutritional counseling and } \\
\text { supplementation }\end{array}$ & & $\begin{array}{l}\text { Identify causes of decreased nutritional } \\
\text { intake; patient education; offer treatments } \\
\text { geared to individual patient }\end{array}$ \\
\hline \multicolumn{3}{|l|}{ Orexigenic agents } \\
\hline Dronabinol & $2.5 \mathrm{mg}$ po BID & $\begin{array}{l}\text { May cause confusion, dizziness, and } \\
\text { dysphoria }\end{array}$ \\
\hline
\end{tabular}

Abbreviations: po, per os (by mouth); QD, once per day; BID, twice per day; TID, 3 times per day; QID, four times per day; LBM, lean body mass. ${ }^{*}$ Dexamethasone is steroid of choice. Administer as a single morning dose with breakfast to reduce possible insomnia. Start with 1-week trial to assess effect. Use prophylactic histamine-2 receptor antagonists to prevent gastric ulceration. Prescribe doses for morning to help avoid insomnia. Be alert for long-term effects, such as weakness, delirium, osteoporosis, and immunosuppression.

${ }^{\dagger}$ For megestrol, start with $160 \mathrm{mg}$ total doses and titrate the dose upward, with optimal dose usually $200 \mathrm{mg}$ po QID (800 mg/day). Be alert for possible increase in thromboembolic events, peripheral edema, breakthrough uterine bleeding, hyperglycemia, and hypertension.

ration of their nutritional status or QOL, as measured by body weight, triceps skinfold measurements, and QOL survey. ${ }^{37}$

Megestrol acetate therapy is best started after adequate enteral nutrition is ensured; side effects may include loss of libido, headache, and rare thrombophlebitis. ${ }^{2}$ It may cause adrenal suppression, and empirical stress doses of glucocorticoids should be considered before surgery or during an acute illness in patients receiving chronic megestrol acetate therapy. It may cause acute hypercalcemia in patients with bone metastasis and has been associated with hyperglycemia. ${ }^{2}$

Another orexigenic agent, dronabinol (delta-9tetrahydrocannabinol) is a synthetic cannabinoid that has important antiemetic properties. ${ }^{33,38}$ No increase in weight was seen when administered to patients with advanced cancer, but increased mood and appetite were noted. ${ }^{38}$ The side effect profile of dronabinol includes dysphoria, confusion, and dizziness. In practice guidelines on the use of appetite stimulants in oncology patients developed by the French National Federation of Cancer Centres, dronabinol was found to have level $\mathrm{C}$ evidence, indicating that the methodology of available studies is too weak, or their results are not consistent when considered together to support its use. ${ }^{39}$ Cyproheptadine, metoclopramide, nandrolone, and pentoxifylline are also being evaluated as orexigenic agents, but currently there is level $\mathrm{C}$ evidence as well. ${ }^{39} \mathrm{It}$ is recommended that these agents be used in combination with nutritional management in the manage- ment of cachexia. In symptomatic patients with incurable cancer of any tumor type, their careful use appears to be warranted. ${ }^{39}$

Omega-3 polyunsaturated fatty acids (n-3 PUFAs) have received considerable attention in the treatment of cancer cachexia. In both animal models and in humans, n-3 PUFAs modulate levels of proinflammatory cytokines, hepatic acutephase proteins, eicosanoids, and tumor-derived factors. They inhibit production of the tumor toxohormones PIF and $\mathrm{LMF}^{35,40}$ and block ubiquitin/ proteosome-induced muscle proteolysis. One n-3 PUFA in particular that has received considerable attention is eicosapentaeonoic acid (EPA), an $\alpha-3-$ omega fatty acid found in marine products such as fish oil. This has been added to nutritional supplements and evaluated in several clinical trials, especially in pancreatic cancer patients. ${ }^{41,42}$ In a study of 31 patients with head and neck cancer experiencing weight loss, a protein and energydense oral supplement containing EPA was found to improve LBM. (Dr. Anne Voss, personal communication). EPA may also suppress the production of IL-6 in patients with pancreatic cancer cachexia. ${ }^{43}$

Despite initial reports in favor of EPA supplementation in patients with cancer cachexia, negative results have been found in 2 placebo-controlled randomized clinical trials. ${ }^{44,45}$ In 1 of these studies, 200 patients with cachexia due to advanced pancreatic cancer were supplemented with either EPA ( $2 \mathrm{~g}$ /day of fish oil in 2 cans of nutritional supplement) or an isocaloric isonitrogenous 
control supplement. ${ }^{44}$ There was no significant difference in weight gain between the 2 groups. In a second clinical trial, 91 patients with a variety of cancer types and loss of both weight and appetite received fish oil $(1000 \mathrm{mg})$ or placebo. ${ }^{45}$ Of the patients who completed the study, supplemental fish oil did not affect appetite, weight loss, caloric intake, nutritional status, or well-being. These studies are complicated by compliance issues, and the true effects of these supplements may be seen once patient compliance is improved. ${ }^{45}$

Another important concept that is gaining acceptance as a possible treatment modality for cachexia is pharmaconutritional support, which is also called nutraceuticals. In a nonrandomized phase II study in which $50 \%$ of patients had head and neck cancer, patients were given supplements including n-3 fatty acids, antioxidant vitamins A and $\mathrm{E}$, and the selective cyclooxygenase (COX-2) inhibitor celecoxib. Whereas only a small number of enrolled patients ${ }^{14}$ received combined treatment with all 3 agents, the authors noted an increase in LBM and body weight, and a decrease in inflammatory cytokine levels in the combined treatment group. These changes were associated with an increased QOL in these patients. This treatment regimen appeared to be safe with maximal compliance. ${ }^{6}$

Perhaps the best way to suppress cancer cachexia is to cure the patient of cancer. Unfortunately, this is not possible for more than $50 \%$ of patients with advanced-stage head and neck cancer. Treating a patient's cachexia during active chemoradiotherapy may increase survival, but treating cachexia may also have significant benefits for the patient who is not being treated for cancer with curative intent. ${ }^{46}$ The patient's weight loss may have emotional implications for the patient, who may experience guilt over not eating, as well as for the family and the caregivers. ${ }^{46}$ For these reasons, treating cancer cachexia makes for both good oncologic and palliative care (Table 4). Currently, the standard agents used to treat cancer cachexia are corticosteroids (eg, dexamethasone) and progestational agents (eg, megestrol acetate) (Table 4).

\section{FUTURE STUDIES}

A growing body of preclinical and clinical evidence suggests that the nonsteroidal antiinflammatory drugs (NSAIDs) may play a role in preventing, and possibly reversing, cachexia. NSAIDs inhibit cyclooxygenase isoenzymes commonly referred to as COX-1 and COX-2. Most NSAIDs are predomi- nantly COX-1 selective, but less gastrointestinal toxicity is seen with COX-2-selective NSAIDs, making COX-2 inhibitors attractive for shortterm use. In a preclinical animal model, cachexia did not develop in athymic mice that had received human HNSCC xenotransplants treated with the COX-2 inhibitor celecoxib, as measured by body weight. Cachexia that developed in mice not initially treated with celecoxib was reversed upon subsequent administration of celecoxib. This finding was correlated with significant reduction in COX-2-derived prostaglandins in tumors. ${ }^{47}$ Two other COX inhibitors, indomethacin and ibuprofen, preserved muscle mass in mice bearing the colon-26 adenocarcinoma, a tumor known to cause significant cancer-associated muscle wasting. ${ }^{48}$ In a retrospective case control analysis of 702 patients with stage IV cancer, long-term ( $>6$ months) use of the COX-1-selective inhibitor indomethacin was associated with weight gain and decreased REE. ${ }^{49}$ More research is currently being conducted to determine the efficacy of NSAIDs in treating cancer cachexia.

Cytokines are a likely mediator of the pathophysiology of cachexia. Cytokine antagonists are being investigated as targeted therapy for possible treatment of cachexia. In murine models, IL-6 antagonists appear to inhibit cancer cachexia. ${ }^{50,51}$ However, the presence of serum IL-6 is not ubiquitous in patients with cachexia, ${ }^{52}$ and it is possible that only a subset of tumors secrete or induce IL-6 at levels high enough to induce cachexia. Thus, serum IL-6 measurements in cancer patients may identify those patients who could benefit from this directed therapy. TNF- $\alpha$ agonists such as etanercept have been shown to decrease levels of IL-6 in cancer patients and may be a potential agent for use in selected patients with cachexia. ${ }^{53}$

Activins, which are members of the transforming growth factor- $\beta$ (TGF- $\beta$ ) superfamily, regulate many diverse activities via serine kinase-mediated signaling. Dysregulation in the activin pathway has been proposed as a potential mechanism in the pathogenesis of cancer cachexia. ${ }^{54}$ A more complete understanding of extracellular activin signaling may inform clinicians how to better treat cancer cachexia.

Anti-cachexia agents that have been successfully used to treat gastrointestinal and other cancers may be considered for future studies with patients with head and neck cancer. Table 5 lists many of the anti-cachexia agents used in human clinical trials to date. Thalidomide, for example, inhibits the production of TNF- $\alpha$ by human 
Table 5. Agents used in cancer cachexia human trials. $^{35,40,41,49-51,53,54}$

\begin{tabular}{|c|c|}
\hline Agent & Type of cancer \\
\hline $\begin{array}{l}\text { Fish oil/omega-3 fatty acids, } \\
\text { eicosapentaenoic acid }\end{array}$ & $\begin{array}{l}\text { Pancreatic cancer, other } \\
\text { malignancies }\end{array}$ \\
\hline Thalidomide & Esophageal cancer \\
\hline Adenosine $5^{\prime}$-triphosphate & Lung cancer \\
\hline Clarithromycin & $\begin{array}{l}\text { Unresectable non-small cell } \\
\text { lung cancer }\end{array}$ \\
\hline Dronabinol & Many malignancies \\
\hline $\begin{array}{l}\text { Erythropoeitin + } \\
\text { Indomethacin }\end{array}$ & Gastrointestinal malignancies \\
\hline Melatonin & Solid tumors \\
\hline Pentoxifylline & Many malignancies \\
\hline $\begin{array}{l}\text { Branched-chain } \\
\text { amino acids }\end{array}$ & $\begin{array}{l}\text { Intra-abdominal } \\
\text { adenocarcinomas }\end{array}$ \\
\hline Hydrazine sulfate & Many malignancies \\
\hline Serotonergic blockade & $\begin{array}{l}\text { Prostate, pancreas, non-small } \\
\text { cell lung cancer }\end{array}$ \\
\hline Carnitine & $\begin{array}{l}\text { Stomach, liver, colon, pancreas, } \\
\text { renal, lung }\end{array}$ \\
\hline $\begin{array}{l}\text { Recombinant IL-2 + } \\
\text { antioxidants }\end{array}$ & Mixed malignancies \\
\hline Ibuprofen & Pancreas \\
\hline Indomethacin & Solid tumors \\
\hline
\end{tabular}

Abbreviation: IL-2, interleukin-2.

macrophages, among other actions. In a recent study of 11 patients with esophageal cancer and cachexia, it reversed the loss of weight and LBM over a 2 -week trial period. ${ }^{55}$ However, peripheral neuropathy, thrombosis formation, and teratogenic effects are some of the possible limiting side effects of thalidomide. Thus, when considering thalidomide and other agents for the treatment of cachexia, potential toxicities should be weighed carefully against potential benefits.

The anabolic effects of testosterone, growth hormone $(\mathrm{GH})$, and insulin-like growth factor-1 (IGF-1) make them potential therapeutic tools. Anabolic steroids such as testosterone are effective in reversing wasting in other catabolic conditions such as human immunodeficiency virus infection, but use in cachexia remains controversial. The possible effects on the tumor must be carefully determined. Administration of testosterone and synthetic anabolic/androgenic steroids may increase LBM, but these agents are mostly used in men because of virilizing effects. ${ }^{56}$

GH and IGF-1 have well-known anabolic effects, but enthusiasm for these agents waned after some large studies revealed negative effects when given to patients in intensive case units. ${ }^{57}$ Ghrelin, a 28-amino acid growth hormone-releasing peptide produced by the stomach, acts to stimulate appetite and the release of growth hormone from the pituitary gland. When administered, it induces increased food intake and an increase in growth hormone, which promotes nutrient incorporation into muscle and fat reserves. Ghrelin may also have an antiinflammatory effect. Therefore, ghrelin may have both GH-dependent and $\mathrm{GH}$-independent mechanisms. Analogs of ghrelin are being tested in preclinical studies to determine the metabolic effects, plasma stability, and half-life. ${ }^{58}$ The effects of these agents not only on the host, but also on the tumor, must be determined with careful preclinical and phase I/II trials to study their possible utility in treating cachexia.

\section{DISCUSSION}

In summary, cancer cachexia is the result of abnormal metabolism that leads to decreased skeletal muscle protein synthesis and increased proteolysis, anorexia, and increased lipolysis that may be induced by many tumor-secreted and host-secreted factors. The metabolic derangement of cachexia causes a severe catabolic state that is exacerbated by a significant systemic inflammatory response. Therefore, the pathophysiology of cancer cachexia is quite different from simple starvation. Successful treatment of this syndrome may require not only nutritional counseling and supplementation but also treatment with anti-cachexia agents to reverse the proteolysis, lipolysis, anorexia, acute-phase response, and inappropriately elevated resting energy expenditure.

Acknowledgments. The authors acknowledge the expert technical support given by the University of North Carolina General Clinical Research Center Grant \#RR000046 (protocol 2254) and the Doris Duke Clinical Research Fellowship Program (JG). The authors also acknowledge the helpful advice given by Dr. Anne Voss, Senior Research Scientist, Ross Products Division, Abbott Laboratories.

\section{REFERENCES}

1. Lees J. Incidence of weight loss in head and neck cancer patients on commencing radiotherapy treatment at a regional oncology center. Eur J Cancer Care 1999;8:133136.

2. Buntzel J, Kuttner $\mathrm{K}$. Value of megestrol acetate in treatment of cachexia in head-neck tumors. Laryngorhinootologie 1995;74:504-507.

3. Tisdale MJ. Cachexia in cancer patients. Nature 2002;2: $862-871$. 
4. Bruera E. ABC of palliative care. Anorexia, cachexia and nutrition. BMJ 1997;315:1219-1222.

5. Inui A. Cancer anorexia-cachexia syndrome: current issues in research and management. CA Cancer J Clin 2002;52: 72-91.

6. Mantovani G, Mededdu C, Maccio A, et al. Cancerrelated anorexia/cachexia syndrome and oxidative stress: an innovative approach beyond current treatment. Cancer Epidermiol Biomarkers Prev 2004;13:1651-1659.

7. Ravasco P, Monteiro-Grillo I, Camilo ME. Does nutrition influence quality of life in cancer patients undergoing radiotherapy? Radiother Oncol 2003;67:213-220.

8. Kim HL, Han K, Zisman A, Figlin RA, Belldegrun AS. Cachexia-like symptoms predict a worse prognosis in localized T1 renal cell carcinoma. J Urol 2004;171:18101813.

9. Andreyev HJ, Norman AR, Oates J, Cunningham D. Why do patients with weight loss have a worse outcome when undergoing chemotherapy for gastrointestinal malignancies? Eur J Cancer 1998;34:503-509.

10. Dewys WD. Weight loss and nutritional abnormalities in cancer patient: incidence, severity, and significance. In: Calmar KC, Fearon KCH, editors. Nutritional support for the cancer patient. London: WB Saunders. p 251-261.

11. Inagaki J, Rodriquez V, Bodey GP. Causes of death in cancer patients. Cancer 1974;33:568-571.

12. Dewys WD, Begg C, Lavin PT, et al. Prognostic effect of weight loss prior to chemotherapy in cancer patients. Am J Med 1980;69:491-497.

13. Guttridge DC, Mayo MW, Madrid LV, Wang C, Baldwin AS. NF-кB-induced loss of MyoD messenger RNA: possible role in muscle decay and cachexia. Science 2000; 289:2363-2366.

14. Kwak KS, Zhou X, Solomon V, et al. Regulation of protein catabolism by muscle-specific and cytokine-inducible ubiquitin ligase E3alplha-II during cancer cachexia. Cancer Res 2004;64:8193-8198.

15. Wyke SM, Tisdale MJ. NF-kappa B mediates proteolysisinducing factor induced protein degradation and expression of the ubiquitin-proteasome system in skeletal muscle. Br J Cancer 2005;92:711-721.

16. Halaas JL. Leptin and the regulation of body weight in mammals. Nature 1998;395:763-770.

17. Chance WT, Balasubramaniam A, Thompson H. Assessment of feeding response of tumor-bearing rats to hypothalamic injection and infusion of neuropeptide Y. Peptides 1996;17:797-801.

18. Gayle D, Ilyin SE, Plata-Salaman CR. Central nervous system IL-1beta system and neuropeptide Y mRNA during IL-1beta-induced anorexia in rats. Brain Res Bull 1997;44:311-317.

19. Acharyya S, Ladner KJ, Nelsen LL, et al. Cancer cachexia is regulated by selective targeting of skeletal muscle gene products. J Clin Invest 2004;114:370-378.

20. Zhou W, Jiang Z, Tian J, Jiang J, Li N, Li J. Role of NF$\kappa \mathrm{B}$ and cytokine in experimental cancer cachexia. World J Gastroenterol 2003;9:1567-1570.

21. Mendell JR, Engel WK. The fine structure of type II muscle fiber atrophy. Neurology 1971;21:358-365.

22. Tomlinson BE, Walton JN, Rebeiz JJ. The effects of ageing and of cachexia upon skeletal muscle. A histopathological study. J Neurol Sci 1969;9:321-346.

23. Mahmoud FA, Rivera NI. The role of C-reactive protein as a prognostic indicator in advanced cancer. Curr Oncol Rep 2002;4:250-255.

24. Sarhill N, Mahmoud FA, Christie R, Tahir A. Assessment of nutritional status and fluid deficits in advanced cancer. Am J Hosp Palliat Care 2003;20:465-473.

25. Bauer JD, Capra S. Nutrition intervention improves outcomes in patients with cancer cachexia receiving chemotherapy-a pilot study. Support Care Cancer 2005;13: 270-274.
26. Fang F, Liu Y, Tang Y, Wang C, Ko S. Quality of life as a survival predictor for patients with advanced head and neck carcinoma treated with radiotherapy. Cancer 2004; 100:425-432.

27. Petruson KM, Silander EM, Hammerlid EB. Quality of life as predictor or weight loss in patients with head and neck cancer. Head Neck 2005;27:302-310.

28. Kim HL, Han K, Zisman A, Figlin RA, Bellidegrun AS. Cachexia-like symptoms predict a worse prognosis in localized T1 renal cell carcinoma. J Urol 2004;171:18101813.

29. Nguyen TV, Yueh B. Weight loss predicts mortality after recurrent oral cavity and oropharyngeal carcinomas. Cancer 2002;95:553-562.

30. Logemann JA, Pauloski BR, Rademaker AW, et al. Xerostomia: 12-month changes in saliva production and its relationship to perception and performance of swallow function, oral intake, and diet after chemoradiation. Head Neck 2003;25:432-437.

31. Kotler DP. Cachexia. Ann Intern Med 2000;133:622634.

32. Russell ST, Tisdale MJ. The role of glucocorticoids in the induction of zinc-alpha $a_{2}$-glycoprotein expression in adipose tissue in cancer cachexia. Br J Cancer. 2005;92:876-881.

33. Von Roenn JH, Paice JA. Control of common, non-pain cancer symptoms. Semin Oncol 2005;32:200-210.

34. Stitt TN, Drujan D, Clarke BA, et al. The IGF-1/PI3K Akt pathway prevents expression of muscle atrophyinduced ubiquitin ligases by inhibiting FOXO transcription factors. Mol Cell 2004;14:395-403.

35. Maltoni M, Nanni O, Scarpi E, Rossi D, Serra P, Amadori D. High-dose progestins for the treatment of cancer anorexia-cachexia syndrome. A systematic review of randomized clinical trials. Ann Oncol 2001;12:289-300.

36. Mantovani G, Maccio A, Bianchi A, et al. Megestrol acetate in neoplastic anorexia/cachexia: clinical evaluation and comparison with cytokine levels in patients with head and neck carcinoma treated with neoadjuvant chemotherapy. Int J Clin Lab Res 1995;25:135-141.

37. Fietkau R, Riepl M, Kettner H, Hinke A, Sauer R. Supportive treatment with megestrol acetate during radio (chemo)therapy in patients with tumors in the head-neck area. A randomized study. Strahlenther Onkol 1996;172: $162-168$.

38. Nelson K, Walsh D, Deeter P, Sheehan F. A phase II study of delta-9-tetrahydrocannabinol for appetite stimulation in cancer-associated anorexia. J Palliat Care 1994; 10:14-18.

39. Desport JS, Gory-Delabaere G, Blanc-Vincent MP, et al. Practice guideline. Standards, options and recommendations for the use of appetite stimulants in oncology (2000). Br J Cancer 2003;89(suppl 1):S98-S100.

40. Russell ST, Tisdale MJ. Effect of eicoasapentaeonic acid (EPA) on expression of a lipid mobilizing factor in adipose tissue in cancer cachexia. Prostaglandins Leukot Essent Fatty Acids 2005;72:409-414.

41. Moses AWG, Slater C, Preston T, Barber MD, Fearon $\mathrm{KCH}$. Reduced total energy expenditure and physical activity in cachectic patients with pancreatic cancer can be modulated by an energy and protein dense oral supplement enriched with n-3 fatty acids. Br J Cancer 2004; 90:996-1002.

42. Barber MD, Ross JA, Voss AC, Tisdale MJ, Fearon KCH The effect of an oral nutritional supplement enriched with fish oil on weight-loss in patients with pancreatic cancer. Br J Cancer 1999;81:80-86.

43. Wigmore SJ, Fearon KC, Maingay JP, Ross JA. Downregulation of the acute-phase response in patients with pancreatic cancer cachexia receiving oral eicosapentaenoic acid is mediated via suppression of interleukin-6. Clin Sci (Colch) 1997;92:215.

44. Fearon KC, Von Meyenfeldt MF, Moses AG, et al. Effect of a protein and energy dense N-3 fatty acid enriched oral 
supplement on loss of weight and lean tissue in cancer cachexia: a randomized double blind trial. Gut 2003;52:1479.

45. Bruera E, Strasser F, Palmer JL, et al. Effect of fish oil on appetite and other symptoms in patients with advanced cancer and anorexia/cachexia: a double-blind placebo-controlled study. J Clin Oncol 2003;21:129.

46. Hopkins K. Food for life, love and hope: an exemplar of the philosophy of palliative care in action. Proc Nutr Soc 2004;63:427-429.

47. Davis TW, Zweifel BS, O’Neal JM, et al. Inhibition of cyclooxygenase-2 by Celecoxib reverses tumor-induced wasting. J Pharm Exp Ther 2004;308:929-934.

48. Lundholm K, Daneryd P, Korner U, Hyltander A, Bosaeus I. Evidence that long-term COX-treatment improves energy homeostasis and body composition in cancer patients with progressive cachexia. Int J Oncol 2004; 24:505-512.

49. Zaki MH, Nemeth JA, Trikha M. CNTO 328, a monoclonal antibody to IL-6, inhibits human tumor-induced cachexia in nude mice. Int J Cancer 2004;111:592-595.

50. McCarthy DO, Whitney P, Hitt A, Al-Majid S. Indomethacin and ibuprofen preserve gastrocnemius muscle mass in mice bearing the colon-26 adenocarcinoma. Res Nurs Health 2004;27:174-184.

51. Enomoto A, Rho MC, Fukami A, Hiraku O, Komiyama K, Hayashi M. Suppression of cancer cachexia by 20S,21epoxy-resibufogenin-3-acetate-a novel nonpeptide IL-6 receptor antagonist. Biochem Biophys Res Commun 2004; 323:1096-1102.

52. Jatoi A, Egner J, Loprinzi CL, et al. Investigating the utility of serum cytokine measurements in a multi-institutional cancer anorexia/weight loss trial. Support Care Cancer 2004;12:640-644.

53. Madhusudan S, Foster M, Muthuramalingam SR, et al. A phase II study of etanercept (Enbrel), a tumor necrosis factor alpha inhibitor in patients with metastatic breast cancer. Clin Cancer Res 2004;10:6528-6534.

54. Harrison CA, Gray PC, Vale WW, Robertson DM. Antagonists of activin signaling: mechanisms and potential biological applications. Trends Endocrinol Metab 2005;16:73-78.

55. Khan ZH, Simpson EJ, Cole AT, et al. Oesophageal cancer and cachexia: the effect of short-term treatment with thalidomide on weight loss and lean body mass. Aliment Pharmacol Ther 2003;17:677-686.

56. Schroeder ET, Zheng L, Yarasheski KE. Treatment with oxandrolone and the durability of effects in older men. J Appl Physiol 2004;96:1055-1062.

57. Takala J, Ruokonen E, Webster NR. Increased mortality associated with growth hormone treatment in critically ill adults. N Engl J Med 1999;341:785-792.

58. Rubinfeld J, Handani M, Taylor JE, et al. Novel ghrelin analogs with improved affinity for the $\mathrm{GH}$ secretagogue receptor stimulate $\mathrm{GH}$ and prolactin release from human pituitary cells. Eur J Endocrinol 2004;151:787-795. 